\title{
Effect of Newtonian Cooling and Non-Linear Density Variation on Non-Darcy Convective Heat and Mass Transfer
}

\author{
Anjna Singh \\ Professor, Department of Mathematics, Government Girls P.G. College, Rewa-486001, Madhya Pradesh, India \\ *Corresponding Author: Anjna Singh, Professor, Department of Mathematics, Government Girls P.G. \\ College, Rewa-486001, Madhya Pradesh, India
}

\begin{abstract}
In this paper is to discuss the Non-linear density temperature effect on free convection flow past a vertical porous plate placed in porous medium in the presence of chemical reaction, viscous dissipation and heat source. The equations governing the flow, heat and mass transfer have been solved by fourth order Runge-Kutta-Fehlberg integration scheme. The effects of various parameters on the flow characteristics have been analyzed.
\end{abstract}

Keywords: Non-Darcym, Heat and Mass Transfer, Cooling and Non-Linear density

\section{INTRODUCTION}

Combined heat and mass transfer problems in presence of chemical reaction are of importance in many processes and thus have received considerable amount of attention in recent times. In processes such as drying, distribution of temperature and moisture over agricultural field and groves of fruit trees, damage of crops due to freezing, evaporation at the surface of a water body, energy transfer in a wet cooling tower and flow in a desert cooler, heat and mass transfer occur simultaneously. Many practical diffusive operations involve the molecular diffusion of a species in the presence of chemical reaction within or at the boundary. Therefore, the study of heat and mass transfer with chemical reaction is of great practical importance to engineers and scientists. Chemical reactions can be codified as either heterogeneous or homogeneous processes. This depends on whether they occur at an interface or as a single-phase volume reaction. A reaction is said to be of first order, if the rate of reaction is directly proportional to the concentration itself. In many chemical engineering processes, there does occur the chemical reaction between a foreign mass and the fluid in which the plate is moving. In view of heat and mass transfer and chemical reactions numerous and wide-ranging applications in various fields like polymer processing industry in particular in manufacturing process of artificial film and artificial fibers and in some applications of dilute polymer solution. Many researchers have studied chemical reaction effects on steady MHD flow with combined heat and mass transfer; (Gangadhar [7], Mohammed Ibrahim [16], Rajashekar et al. [19], Kishan and Srinivas [11], Anjalidevi and David [4]. Merkin [15] was the first to consider a somewhat different but practically relevant driving mechanism for the natural convection boundary layer flow near a vertical surface in which it was assumed that the flow was setup by the Newtonian heating from the bounding surface, i.e. the heat transfer from the surface was taken to be proportional to the local surface temperature. The situation with Newtonian heating arises in what are usually termed conjugate convective flows, where the heat is supplied to the convicting fluid through a bounding surface with a finite heat capacity. The steady free convection boundary layer along a semiinfinite plate, slightly inclined to the horizontal and embedded in a porous medium with the flow generated by Newtonian heating was investigated by Lesnic et al. [13].Madhusudhan Rao et al. [14] has studied Soret and Dufour effects on Hydro-Magnetic heat and mass transfer over a vertical plate in a porous medium with a convective surface boundary condition and chemical reaction. Lavanya et al. [12] has studied Dufour and Soret effects on steady MHD free convective flow past a vertical porous plate embedded in a porous medium with chemical reaction heat generation and viscous dissipation. 
Chaudhary and Jain [6] presented an exact solution for the unsteady free convection boundary layer flow of an incompressible fluid past an infinite vertical plate with the flow generated by Newtonian heating and impulsive motion of the plate. Rajesh et al. [20] studied unsteady convective flow past an exponentially accelerated infinite vertical porous plate with Newtonian heating and viscous dissipation.

The study of heat generation in moving fluids is important as it changes the temperature distribution and the particle deposition rate particularly in nuclear reactor cores, fire and combustion modeling, electronic chips and semi conductor wafers. Heat generation is also important in the context of exothermic or endothermic chemical reaction. Vajravelu and Hadjinicolaou [20] studied the heat transfer characteristics in the laminar boundary layer of a viscous fluid over a stretching sheet with viscous dissipation or frictional heating and internal heat generation. Hossain et al [10] studied problem of the natural convection flow along a vertical wavy surface with uniform surface temperature in the presence of heat generation/absorption. Mohammed Ibrahim and Bhaskar Reddy [17] investigated heat and mass transfer effects on steady MHD free convective flow along a stretching surface with dissipation, heat generation and radiation.

But in the above mentioned studies, Dufour and Soret terms have been neglected from the energy and concentration equations respectively. It has been found that energy flux can be generated not only by temperature gradient but also by concentration gradient as well. The energy flux caused by concentration gradient is called Dufour effect and the same by temperature gradient is called the Soret effect. These effects are very significant when the temperature and concentration gradient are very high. Anghel et al. [3] studied the Dufour and Soret effects on free convection boundary layer over a vertical surface embedded in a porous medium. Postelnicu [18] analyzed the influence of magnetic field on heat and mass transfer from vertical surfaces in porous media considering Soret and Dufour effects. Alam et al. [1] investigated the Dufour and Soret effects on steady MHD mixed convective and mass transfer flow past a semi-infinite vertical plate. Chamkha and Ben-Nakhi [5] analyzed MHD mixed convectionradiation interaction along a permeable surface immersed in a porous medium in the presence of Soret and Dufour effects. M.S. Alam and M.M Rahman [2] investigated Chemical reaction and radiation effects on MHD free convection flow along a stretching surface with viscous dissipation and heat generation.

In all the above a linear density variations is considered in the equation of state. This is valid for temperature variation at $20^{\circ} \mathrm{C}$. But this analysis is not applicable to the study of the flow of water at $4^{0} \mathrm{C}$. The density of water is Maximum at atmospheric pressure and the modified form of the equation to water at $4^{0} \mathrm{c}$ is given by $\Delta \rho=-\rho \gamma(\Delta \mathrm{T})^{2}$

Where $\gamma=8 \times 10^{-6}(0 \mathrm{c})^{-2}$. Taking this fact into account, Goren [8] showed in this case similarity solution for the free convection flow of water at $4^{0} \mathrm{c}$ past a semi-finite vertical plate exists. Govindarajulu [9] showed that a similarity solution exists for the free convection flow of water at $4^{\circ} \mathrm{C}$ from vertical and horizontal plates in the presence of suction and injection. Several authors have investigated the effect of non-uniform density-temperature relation on convective heat/mass transfer problems.

\section{Formulation of the Problem}

A steady two-dimensional flow of an incompressible viscous fluid, along an infinite vertical porous plate embedded in a porous medium is considered. The $x$ - axis is taken on the infinite plate, and parallel to the free-stream velocity which is vertical and the $y$-axis is taken normal to the plate. Initially the plate and the fluid are at same at temperature $\mathrm{T}_{\infty}$ in a stationary condition with concentration level $\mathrm{C}_{\infty}$ at all points. The plate starts moving impulsively in its own plane with velocity $U_{0}$, its temperature is raised to $T_{w}$ and the concentration level at the plate is raised to $C_{w}$. A homogeneous first order chemical reaction between fluid and the species concentration is considered, in which the rate of chemical reaction is directly proportional to the species concentration. The flow configuration and coordinate system are shown in the Figure 1. 


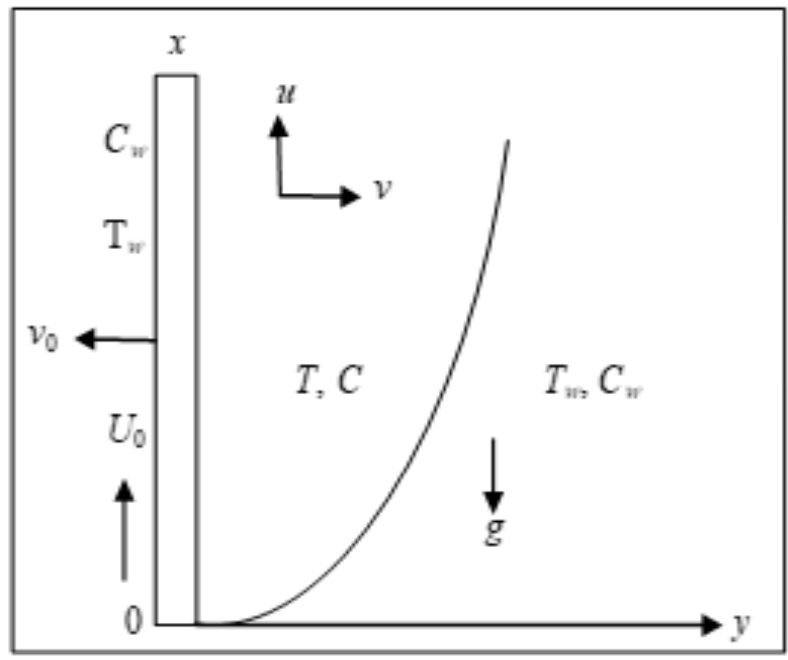

Fig1. Flow configuration and coordinate system

The fluid is considered to be a gray, absorbing emitting radiation but non-scattering medium and the Rosseland approximation is used to describe the radiative heat flux in the energy equation. It is also assumed that all the fluid properties are constant except that of the influence of the density variation with temperature and concentration in the body force term (Boussinesq approximation). Then, under the above assumptions, the governing boundary layer equations are

\section{Continuity equation}

$\frac{\partial u}{\partial x}+\frac{\partial v}{\partial y}=0$

\section{Momentum equation}

$u \frac{\partial u}{\partial x}+v \frac{\partial u}{\partial y}=v \frac{\partial^{2} u}{\partial y^{2}}+g \beta_{0}\left(T-T_{\infty}\right)+g \beta_{1}\left(T-T_{\infty}\right)^{2}+\beta^{\bullet}\left(C-C T_{\infty}\right)-\left(\frac{v}{k}\right) u-\left(\frac{b}{k}\right) u^{2}$

\section{Energy equation}

$u \frac{\partial T}{\partial x}+v \frac{\partial T}{\partial y}=\alpha \frac{\partial^{2} T}{\partial y^{2}}+\frac{D_{m}}{c_{s}} \frac{k_{T}}{c_{p}}+\frac{Q_{0}}{\rho c_{p}}\left(T-T_{\infty}\right)+\frac{\mu}{\rho c_{p}}\left(\frac{\partial u}{\partial y}\right)^{2}$

\section{Concentration equation}

$u \frac{\partial C}{\partial x}+v \frac{\partial C}{\partial y}=D_{m} \frac{\partial^{2} C}{\partial y^{2}}+\frac{D_{m} k_{T}}{T_{m}} \frac{\partial^{2} T}{\partial y^{2}}-k r^{\prime}\left(C-C_{\infty}\right)$

where $\mathrm{u}, \mathrm{v}$ are the Darcian velocities components in the $\mathrm{x}$ and $\mathrm{y}$ directions respectively, $\mathrm{v}$ is the kinematic viscosity, $g$ is the acceleration due to gravity, $\rho$ is the density, $\mu$ is the dynamic viscosity, $\beta_{0} \beta_{1}$ are the coefficients of volume expansion with temperature, $\beta^{*}$ is the volumetric coefficient of expansion with concentration, $\mathrm{b}$ is the empirical constant, $\mathrm{T}, \mathrm{T}_{\mathrm{w}}$ and $\mathrm{T}_{\infty}$ are the temperature of the fluid inside the thermal boundary layer, the plate temperature and the fluid temperature in the free stream, respectively, $\mathrm{C}, \mathrm{C}_{\mathrm{w}}$ and $\mathrm{C}_{\infty}$ are the corresponding concentrations. $\mathrm{K}$ is the Darcy permeability. $\sigma$ is the electric conductivity.

$\alpha=\frac{k_{f}}{\rho_{0} c_{p}}$ is the thermal diffusivity, $\mathrm{c}_{\mathrm{p}}$ is the specific heat at constant pressure. $\mathrm{D}_{\mathrm{m}}$ is the coefficient of mass diffusivity, $k_{T}$ is thermal diffusion ratio, $\mathrm{c}_{\mathrm{s}}$ is the concentration susceptibility, the $Q_{0}\left(T-T_{\infty}\right)$ is assumed to be amount of heat generated or absorbed per unit volume and $Q_{0}$ is a constant, which may take on either positive or negative values, $\quad k r$ 'is chemical reaction parameter.

The boundary conditions for velocity, temperature and concentration fields are given by

$u=U_{0}, v=v_{0}(x),-k_{f} \frac{\partial T}{\partial y}=h\left[T-T_{w}(x, 0)\right], C=C_{W}$ at $\mathrm{y}=0$

$u=0, v=0, T=T_{\infty}, C=C_{\infty}$ at $y \rightarrow \infty$

Where $\mathrm{U}_{0}$ is the uniform velocity and $v_{0}(x)$ is the velocity of suction at the plate...

The equations (2) - (4) are coupled, parabolic and nonlinear partial differential equations and hence 
analytical solution is not possible. Therefore numerical technique is employed to obtain the required solution. Numerical computations are greatly facilitated by non-dimensionalization of the equations. Proceeding with the analysis, we introduce the following similarity transformations and dimensionless variables which will convert the partial differential equations from two independent variables $(\mathrm{x}, \mathrm{y})$ to a system of coupled, non-linear ordinary differential equations in a single variable $(\eta)$ i.e., coordinate normal to the plate. In order to write the governing equations and the boundary conditions in dimensionless form, the following non-dimensional quantities are introduced.

$\eta=y \sqrt{\frac{U_{0}}{2 v x}}, \quad \psi=\sqrt{2 v x U_{0}} \mathrm{f}(\eta)$

$\theta(\eta)=\frac{T-T_{\infty}}{T_{W}-T_{\infty}} \quad \phi(\eta)=\frac{C-C_{\infty}}{C_{W}-C_{\infty}}$

Where $f(\eta)$ is the dimensionless steam function and $\psi$ is the dimensional stream function defined in the usual way

$u=\frac{\partial \psi}{\partial y} \quad$ and $\quad v=-\frac{\partial \psi}{\partial x}$

Cleary the continuity equation (1) is identically satisfied.

Then introducing the relation (6) into equation (1) we obtain

$u=U_{0} f^{\prime}(\eta)$ and $v=\sqrt{\frac{v U_{0}}{2 x}}\left(\eta f^{\prime}-f\right)$

Further introducing equation (6) and (7) in to momentum equation (2), Energy equation (3) and Concentration equation (4) we obtain the following local similarity equations.

$f^{\prime \prime}+f f^{\prime}-\left(\frac{1}{D a R e^{\prime F s^{\prime 2}}}\right.$

$\theta^{\prime \prime}+\operatorname{Pr} f \theta^{\prime}+\operatorname{Pr} D u \phi^{\prime \prime}+\operatorname{Pr} Q \operatorname{Re} \theta+\operatorname{Pr} E c\left(f^{\prime \prime}\right)^{2}=0$

$\phi^{\prime \prime}+\operatorname{Scf} \phi^{\prime}+\operatorname{ScSr} \theta^{\prime \prime}-\operatorname{Kr} \phi=0$

Where

$G r=\frac{g \beta\left(T_{W}-T_{\infty}\right) 2 x}{U_{0}^{2}}$ (Grashof number),$N=\frac{\beta^{*}\left(C_{W}-C_{\infty}\right)}{\beta\left(T_{W}-T_{\infty}\right)}$ (Buoyancy ratio)

$D a=\frac{K}{2 x^{2}}$ (Darcy number), Re $=\frac{U_{0} x}{v}$ (Reynolds number),

$F s=\frac{b}{x}$ (Forchheimer number), $\operatorname{Pr}=\frac{v}{\alpha}$ (Prandtl number)

$D u=\frac{D_{m} K_{T}\left(C_{w}-C_{\infty}\right)}{C_{s} C_{p} v\left(T_{w}-T_{\infty}\right)}$ (Dufour number),Sr $=\frac{D_{m} K_{T}\left(T_{w}-T_{\infty}\right)}{v T_{m}\left(C_{w}-C_{\infty}\right)}$ (Soret number)

$E c=\frac{U_{0}^{2}}{c_{p}\left(T_{w}-T_{\infty}\right)}$ Eckert number), $Q=\frac{Q_{0} v}{\rho c_{P} U_{0}^{2}} \quad$ (Heat generation parameter),

$S c=\frac{v}{D_{m}} \quad$ (Schmidt number),$\gamma=\frac{\beta_{1} \Delta T}{\beta_{0}} \quad$ (Density ratio),

$K r=\frac{2 K_{r}^{\prime} v x}{D_{m} U_{0}} \quad$ (Chemical reaction parameter), $B i=\left(\frac{h}{k}\right) \sqrt{\frac{2 v x}{U_{0}}}$ (Convective Heat transfer parameter)

The corresponding boundary conditions are

$$
\begin{aligned}
& f=f_{w}, f^{\prime}=1,, \phi=1 \text { at } \eta=0 \quad \theta^{\prime}(0)=B i[\theta(0)-1] \\
& f^{\prime}=0, \theta=0, \phi=0 \text { at } \eta \rightarrow \infty
\end{aligned}
$$

Where $f_{w}=-v_{0} \sqrt{\frac{2 x}{v U_{0}}}$ the dimensionless suction velocity and primes is is denote partial differentiation with respect to the variable $\eta$.

\section{Skin Friction, NuSSELt Number AND SHERWOOd Number}

The parameters of engineering interest for the present problem are the skin-friction coefficient, the Nusselt number and the Sherwood number, which are given respectively by the following expressions. Knowing the velocity field the skin-friction at the plate can be obtained, which in non-dimensional form is given by 
$\frac{1}{2} R e^{\frac{1}{2}} C_{f}=f^{\prime \prime}(0)$

Knowing the temperature field, the rate of heat transfer coefficient can be obtained, which in nondimensional form in terms of Nusselt number, is given by

$N u e^{\frac{1}{2}}=-\theta^{\prime}(0)$

Knowing the concentration field, the rate of mass transfer coefficient can be obtained, which in nondimensional form, in terms of Sherwood number, is given by

$\operatorname{Sh} \operatorname{Re}^{\frac{1}{2}}=\phi^{\prime}(0)$

Where $R e=\frac{U_{0} x}{v}$ is the Reynolds Number

\section{COMPARISON}

Comparison values of the Skin friction, the rate of heat and mass transfer at the plate $\eta=0$ is shown in the table 1. In the case of Linear density temperature relation $(\gamma=0), \mathrm{Cf}, \mathrm{Nu}$ and $\mathrm{Sh}$ are in good agreement with Alam et al. [2] with $\mathrm{G}=12, \mathrm{M}=0, \mathrm{f}_{\mathrm{w}}-0.5, \mathrm{~N}=1, \mathrm{Da}^{-1}=1 \mathrm{Pr}=0.71, \mathrm{~F}_{\mathrm{s}=1} \mathrm{Re}=100$ and $\mathrm{Sc}=0.22$.

TABLE1.

\begin{tabular}{|c|c|c|c|c|c|c|c|}
\hline \multicolumn{2}{|c|}{ Parameters } & \multicolumn{2}{c|}{$\mathbf{C f}_{\mathbf{x}}$} & \multicolumn{2}{c|}{ Nu } & \multicolumn{2}{c|}{ Sh } \\
\hline Sr & Du & $\begin{array}{c}\text { Alam \& } \\
\text { Rahman }\end{array}$ & Present & $\begin{array}{c}\text { Alam \& } \\
\text { Rahman }\end{array}$ & Present & $\begin{array}{c}\text { Alam \& } \\
\text { Rahman }\end{array}$ & Present \\
\hline 2.0 & 0.03 & 3.4231141 & 3.42067 & 1.0283189 & 1.027999 & 0.1296854 & 0.129599 \\
\hline 1.0 & 0.06 & 3.3457474 & 3.34456 & 1.0155338 & 1.014999 & 0.2992750 & 0.298996 \\
\hline 0.5 & 0.12 & 3.3162482 & 3.31599 & 1.0019868 & 1.001899 & 0.3844602 & 0.383897 \\
\hline 0.4 & 0.15 & 3.3141130 & 3.31399 & 0.9965224 & 1.995789 & 0.4017999 & 0.401568 \\
\hline 0.2 & 0.30 & 3.3287043 & 3.328567 & 0.9718535 & 0.969985 & 0.4381199 & 0.435508 \\
\hline 0.1 & 0.60 & 3.3828661 & 3.382794 & 0.9248360 & 0.919916 & 0.4602605 & 0.459959 \\
\hline
\end{tabular}

\section{DISCUSSION OF THE NUMERICAL RESULTS}

The numerical solutions of the non-linear differential equations (8) - (10) under the boundary conditions (11) have been performed by applying a shooting technique along with the fourth order Runge-Kutta method. First of all higher order non-linear differential equations (8) - (10) are converted into simultaneous linear differential equations of first order and they are further transformed into initial problem by applying the Shooting method. From this process of numerical computation, the skinfriction coefficient, the Nusselt Number and Sherwood Number which are respectively proportional to $f^{\prime},-\theta^{\prime}(0)$ and $-\varphi^{\prime}(0)$ are also sorted out and their numerical values are presented in a tabular form.

In order to analyze the effects of various physical parameters on the flow, heat and mass transfer of a viscous fluid over a vertical plate, a numerical computation has been performed. The effects of various physical parameters on the velocity profiles, temperature and concentration profiles have been discussed and are shown graphically in figures $2-34$.

Figures 2, 13 and 24 represent the velocity, temperature and concentration with Grashof number Gr. It is found that the magnitude of the velocity enhances with increase in $\mathrm{Gr}$ (fig 2). From fig 14 we find that the temperature reduces with increase in Gr. From fig 26 we find that the concentration enhances with increase in $\mathrm{Gr}$.

Figs 3, 14 and 25 represent $\mathrm{f}^{1}, \theta$, and $\phi$ with magnetic parameter $\mathrm{Da}^{-1}$. The variation of $\mathrm{f}^{1}, \theta$, and $\phi$ with $\mathrm{Da}^{-1}$ shows that lesser the permeability of the porous medium smaller the velocity, temperature and larger the concentration the boundary layer.

Figures 4,15 and 26 represents $\mathrm{f}^{1}, \theta$, and $\phi$ with buoyancy ratio $\mathrm{N}$. It is found that when the molecular buoyancy force dominates over the thermal buoyancy force the velocity and concentration reduce and temperature enhances in the flow region when the buoyancy forces are in the same direction and for the forces acting in opposite direction the velocity enhances while the temperature and concentration reduces in the flow field.Figures5, 16 and 27 represents $\mathrm{f}^{1}, \theta$, and $\phi$ with chemical reaction parameter 
$\mathrm{Kr}$. It can be seen from the profiles that the velocity, temperature and concentration reduces in the case of $\mathrm{Kr}>0$ and for $\mathrm{Kr}<0$, we notice depreciation in $\theta$ and $\phi$ and enhancement in $\mathrm{f}^{1}$ in entire fluid region.

Figures $6,17,28$ depict $\mathrm{f}^{1}, \theta$, and $\phi$ with heat source parameter $\mathrm{Q}$. It is found that an increase in the strength of heat generating /absorption reduces the velocity, temperature and concentration in the boundary layer.

Figs 7, 18, 29 represents $\mathrm{f}^{1}, \theta$, and $\phi$ with Eckert number Ec. Higher the dissipative heat larger the temperature and smaller the velocity and concentration in the boundary layer.

Figures 8, 18, 29 illustrate the effect of Soret and Dufour parameter on the variations of the fluid velocity, temperature and concentration respectively. It is found that increase in the Soret parameter $\mathrm{Sr}$ (or decrease in the Dufour parameter $\mathrm{Du}$ ) reduces the velocity, the temperature and concentration in the boundary layer.

Figures 9, 20, 31 represents $\mathrm{f}^{1}, \theta$, and $\phi$ with convective heat transfer coefficient $\mathrm{Bi}$. It is found that an increase in Bi leads to an enhancement in $\mathrm{f}^{1}, \theta$ and $\phi$.

Figures 10, 21, 32 depict the effect of Forchheimer number Fs on velocity, temperature and concentration. It is observed from fig. 11that the velocity of the fluid decreases with increase in Fs. Since Forchheimer number Fs represent the inertial drag, thus an increase in the Forchheimer number Fs, increases the resistance to the flow and so a decrease in the fluid velocity ensues. It is noticed from figs $23 \& 34$ that the temperature and concentration reduces with increase in Fs.

The effect of suction parameter $f_{w}$ on the velocity profiles is shown in fig 11 . It is found from the figure that the velocity profiles decrease monotonically with the increase in the suction parameter indicating the fact that suction parameter stabilizes the boundary layer growth. The effect of suction parameter on the temperature and concentration is shown in figs 22 and 33. An increase in $\left|f_{w}\right|(<0)$ leads to an enhancement in $\mathrm{f}^{1}$ and depreciation in $\theta$, and $\phi$.

The effect of density ratio $\gamma$ on $\mathrm{f}^{1}, \theta$, and $\phi$ is shown in figs 12, 23, 34. From figs we find that the velocity $\mathrm{f}^{1}, \theta$, and $\phi$ reduces with increase in the density ratio. Thus the non-linearity in the densitytemperature relation results in depreciation in $\mathrm{f}^{1}, \theta$, and $\phi$.

The skin friction $(\tau)$ on the surface $\eta=0$ is shown in table 2 for different values of $\mathrm{N}, \mathrm{Kr}, \mathrm{Q}, \mathrm{Ec}, \mathrm{Sr} \&$ $\mathrm{Du} \mathrm{Bi}, \& \gamma$. With reference to the buoyancy ratio $\mathrm{N}$ we find that when the molecular buoyancy force dominates over the thermal buoyancy force, $|\tau|$ reduces on the wall when the buoyancy forces are in the same direction and for the forces acting in the opposite directions it enhances on the wall. The variation of $\tau$ with the chemical reaction parameter $\mathrm{Kr}$ shows that $|\tau|$ enhances in the degenerating chemical reaction case and reduces in the generating chemical reaction case. An increase in the convective heat transfer coefficient Bi (or) Eckert number Ec reduces $|\tau|$ on the wall. With reference to the heat source parameter $\mathrm{Q}$ we find that $|\tau|$ enhances with increase in the strength of heat generating / absorbing source. Increase in the Soret parameter $S_{\mathrm{r}}$ (or decrease in the Dufour parameter Du) leads to an enhancement in $|\tau|$. An increase in the density ratio $\gamma$ reduces $|\tau|$. Thus the non-linearity in the density-temperature relation leads to a depreciation in $|\tau|$ on the wall.

The rate of heat transfer (Nusselt number) is exhibited in the table 2 for different variation of parameters. With reference to buoyancy ratio $\mathrm{N}$ it is observed that $|\mathrm{Nu}|$ enhances with $|\mathrm{N}|$ irrespective of the directions of the buoyancy forces. $|\mathrm{Nu}|$ enhances on the wall in the degenerating chemical reaction case and reduces in the generating chemical reaction case. An increase in Ec or Bi leads to an enhancement in the rate of heat transfer. The variation of $\mathrm{Nu}$ with chemical reaction parameter $\mathrm{Kr}$ shows that $|\mathrm{Nu}|$ enhances in the degenerating chemical reaction case and reduces in the generating chemical reaction case. $|\mathrm{Nu}|$ enhances on the wall $\eta=0$ with increase in the strength of the heat generating / absorption source. Increase in the soret parameter $\mathrm{Sr}$ (or decrease in $\mathrm{Du}$ ) results an enhancement in $|\mathrm{Nu}|$. An increase in the density ratio $\gamma$ enhances $|\mathrm{Nu}|$. Thus the non-linearity in the density temperature relation leads to an enhancement in the rate of heat transfer on the wall.

The rate of mass transfer (Sherwood Number) on the wall $\eta=0$ is exhibited in the table 2 for different variations. It is found that the rate of mass transfer reduces with increase in convection heat transfer coefficient $\mathrm{Bi}$. With reference to buoyancy ratio $\mathrm{N}$ it is found that the rate of mass transfer reduces when 
the buoyancy forces are in the same direction and for the forces acting in opposite directions it enhances on the wall. Also $|\mathrm{Sh}|$ enhances in the degenerating chemical reaction case and reduces in the generating chemical reaction case.Higher the dissipative heat smaller $|\mathrm{Sh}|$ on the wall. Increasing $\mathrm{Sr}$ (or decreasing $\mathrm{Du})$ results in an enhancement in $|\mathrm{Sh}|$ on the wall. An increase in the density ratio $\gamma$ leads to a depreciation in $|\mathrm{Sh}|$. Then the non linearity in the density-temperature relation leads to a reduction in $|\mathrm{Sh}|$ on the wall.
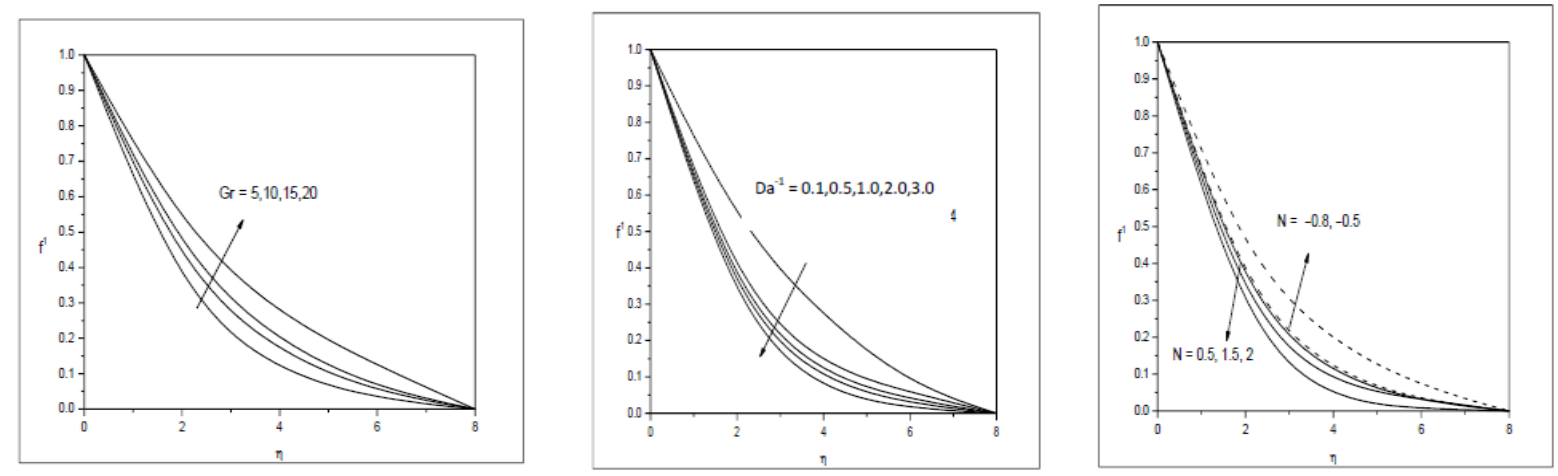

Fig. 2 Variation of velocity (f) with Grashof parameter(G) Fig. 3 Variation of velocity (f) with inverse Darcy parameter)(Da ${ }^{-1}$ ) Fig. 4 Variation of velocity (f) with buoyancy ratio (N)

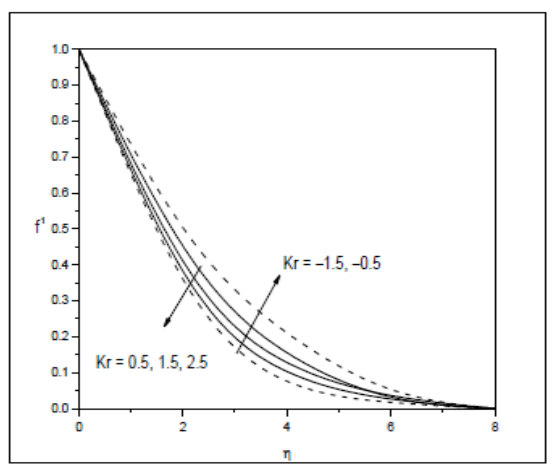

Fig. 5 Velocity profiles (f) with $\mathrm{Kr}$

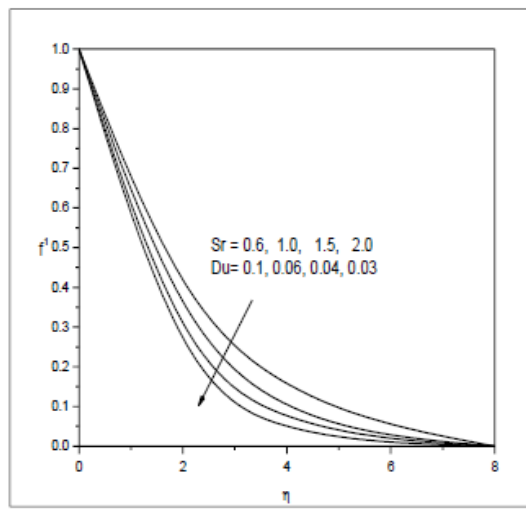

Fig.8 Velocity profiles with $\mathrm{Sr}$ and $\mathrm{Du}$

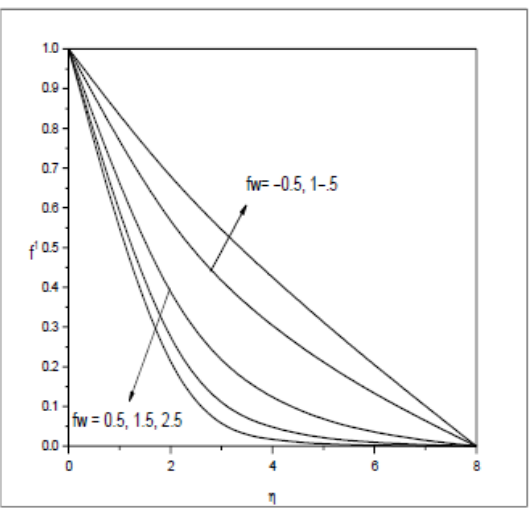

Fig. 11 Velocity profiles (f') with $f_{w}$

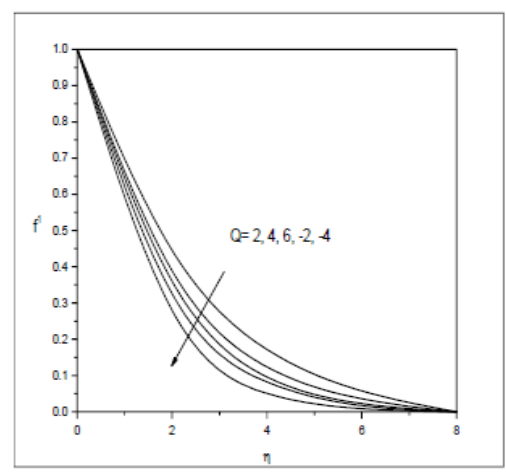

Fig. 6 Velocity profiles (f) with Q

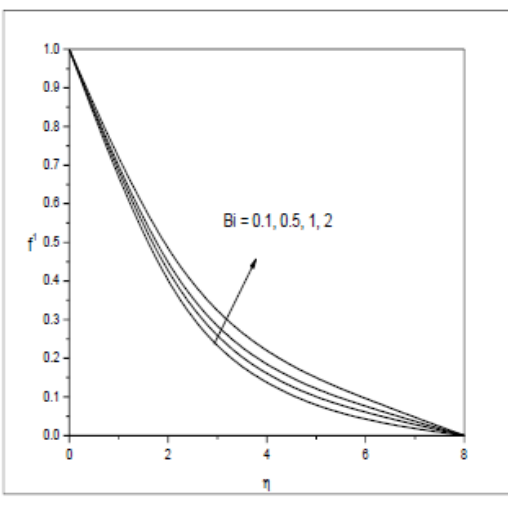

Fig. 9 Velocity profiles (f) with $\mathrm{Bi}$

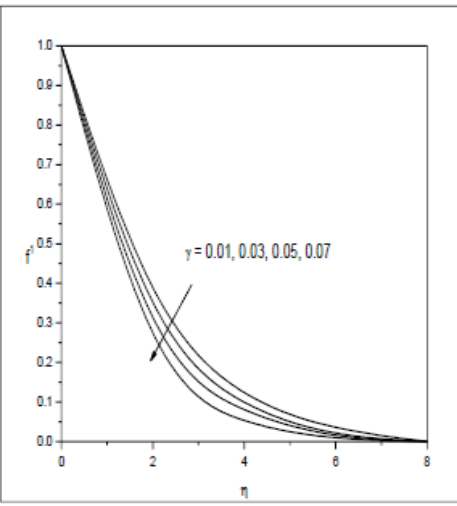

Fig. 12 Velocity profiles (f $)$ with $\gamma$

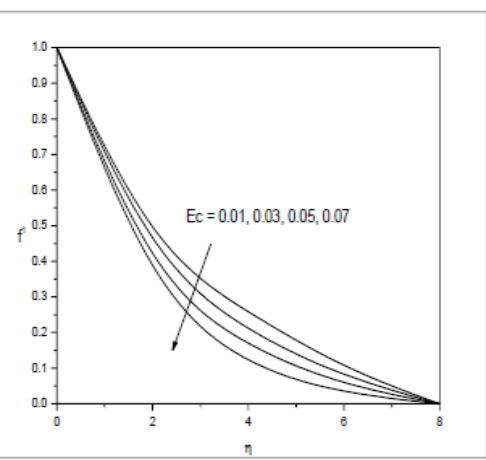

Fig.7 Velocity profiles (f) with Ec

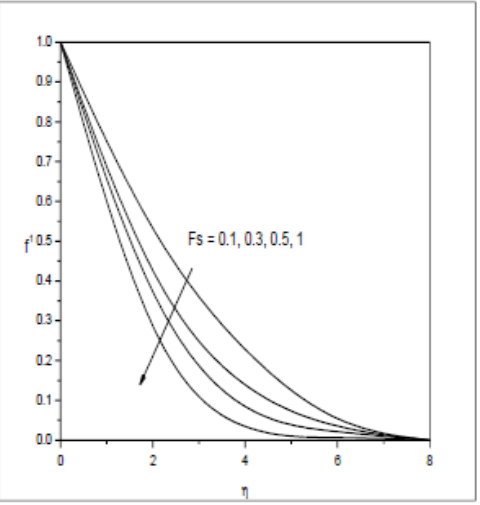

Fig. 10 Velocity profiles (f) with Fs

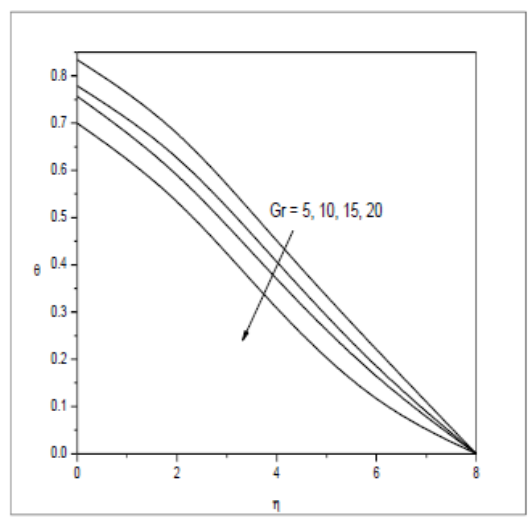

Fig. 13 Temperature profiles $(\theta)$ with $\mathrm{G}$ 
Effect of Newtonian Cooling and Non-Linear Density Variation on Non-Darcy Convective Heat and Mass Transfer

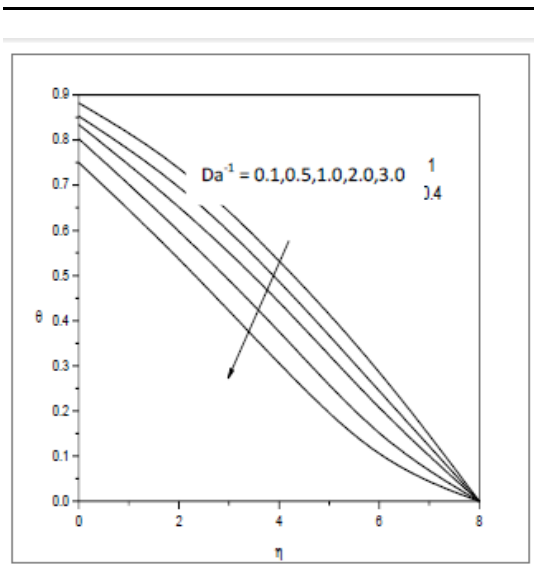

Fig. 14 Temperature profiles $(\theta)$ with $\mathrm{Da}^{-1}$

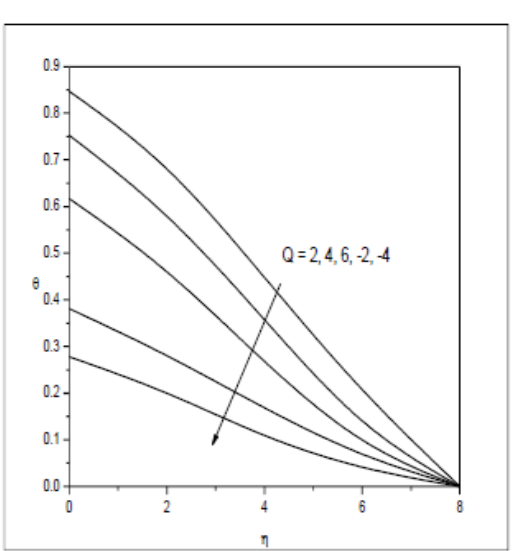

Fig. 17 Temperature profiles $(\theta)$ with $Q$

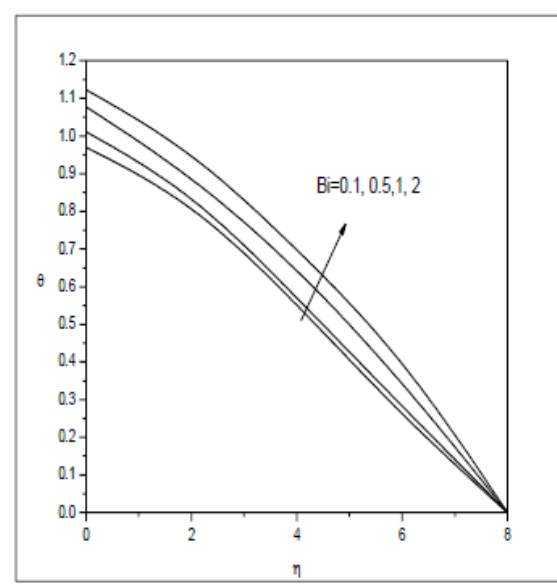

Fig. 20 Temperature profiles $(\theta)$ with $\mathrm{Bi}$

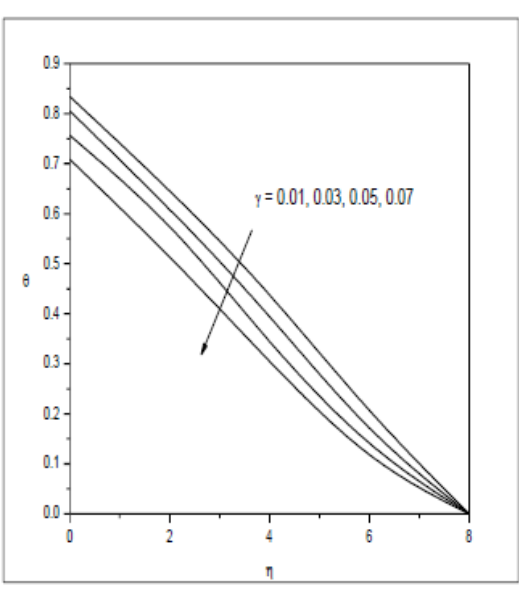

Fig. 23 Temperature profiles $(\theta)$ with $\gamma$

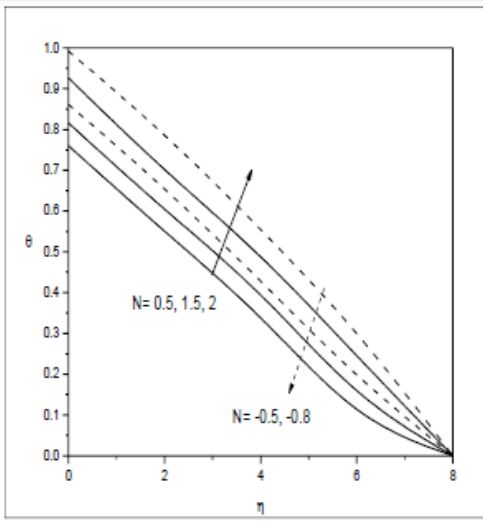

Fig. 15 Temperature profiles $(\theta)$ with $\mathrm{N}$

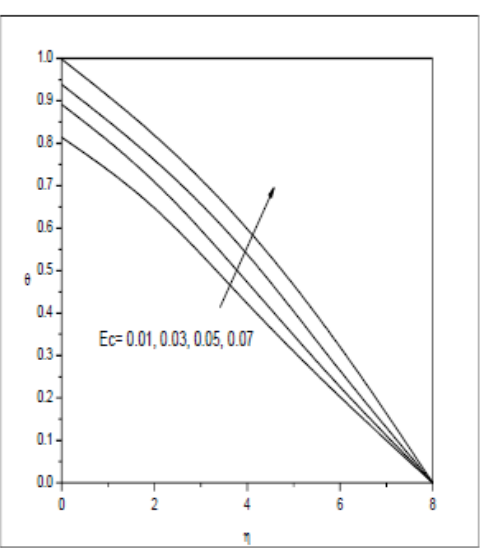

Fig. 18 Temperature profiles $(\theta)$ with Ec

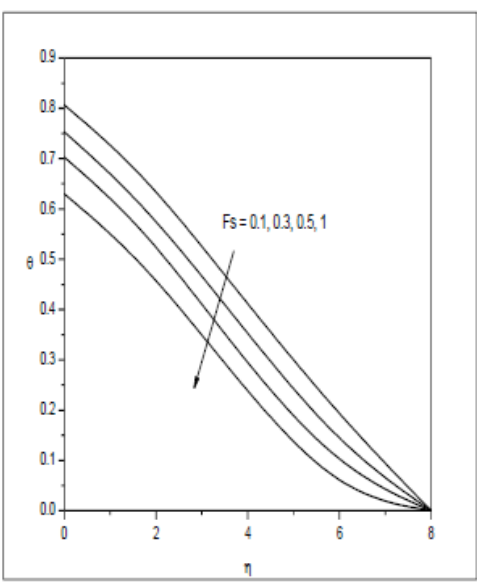

Fig. 21 Temperature profiles $(\theta)$ with Fs

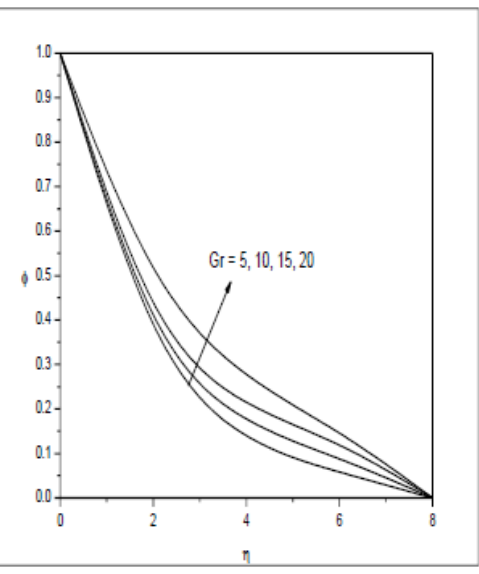

Fig. 24 Concentration profiles ( $\phi)$ with $\mathrm{G}$

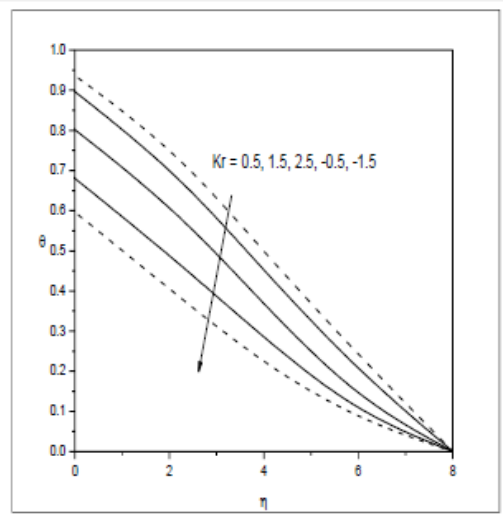

Fig.16 Temperature profiles for different values of $\mathrm{Kr}$

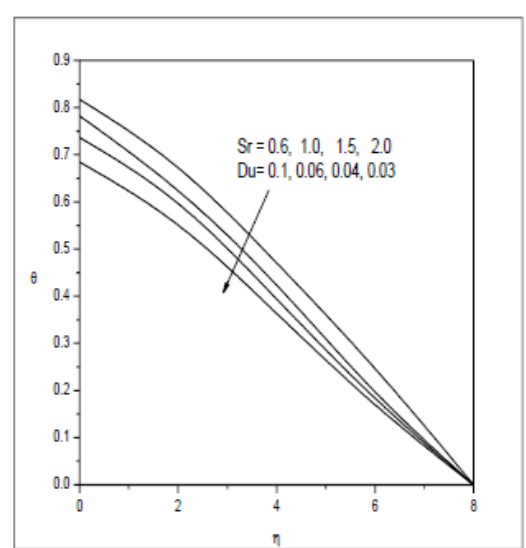

Fig. 19 Temperature profiles $(\theta)$ with Sr \&Du

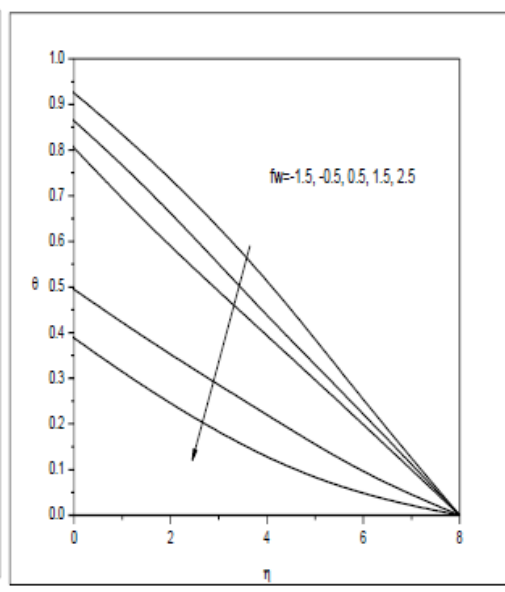

Fig. 22 Temperature profiles $(\theta)$ with $f_{w}$

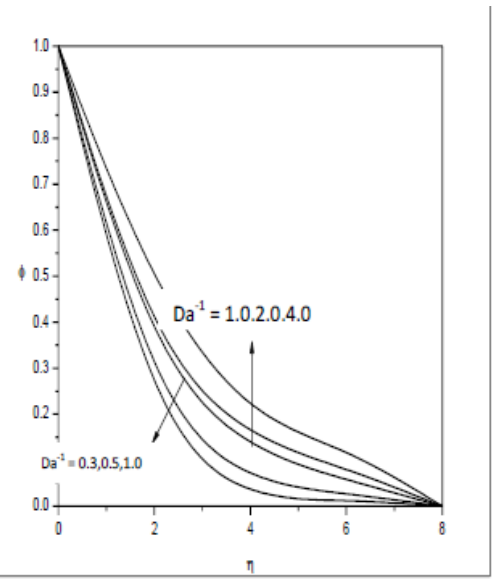

Fig. 25 Concentration profiles $(\phi)$ with $\mathrm{Da}^{-1}$ 
Effect of Newtonian Cooling and Non-Linear Density Variation on Non-Darcy Convective Heat and Mass Transfer

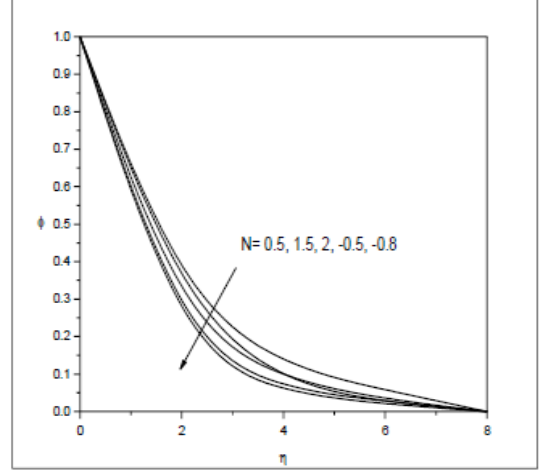

Fig. 26 Concentration profiles $(\phi)$ with $\mathrm{N}$

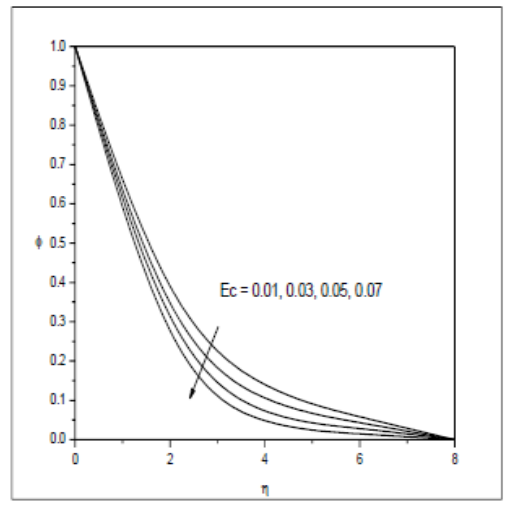

Fig. 29 Concentration profiles ( $\phi$ ) with Eckert number Ec

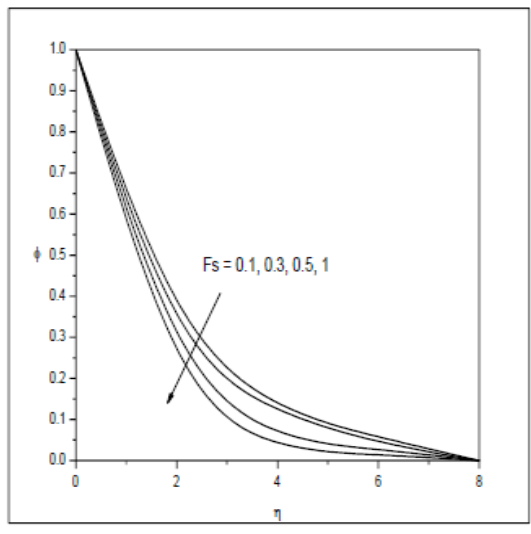

Fig. 32 Concentration profiles ( $\phi)$ with Fs

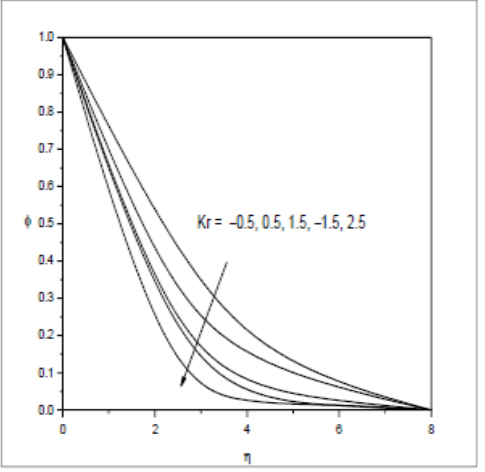

Fig. 27 Concentration profiles $(\phi)$ for different values of $\mathrm{Kr}$

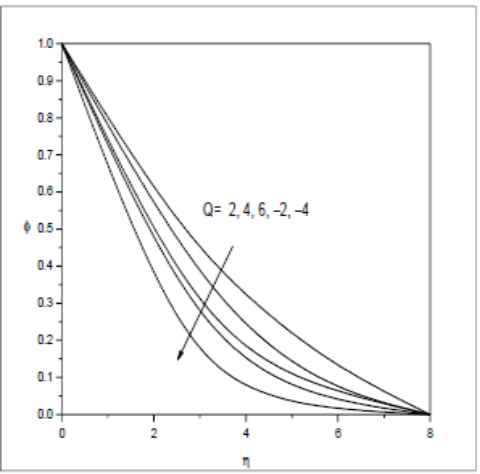

Fig. 28 Concentration profiles $(\phi)$ with $Q$

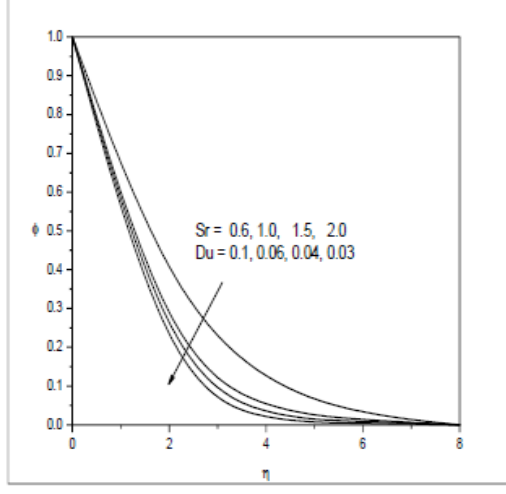

Fig. 30 Concentration profiles $(\phi)$ with $\mathrm{Sr}$ and $\mathrm{Du}$

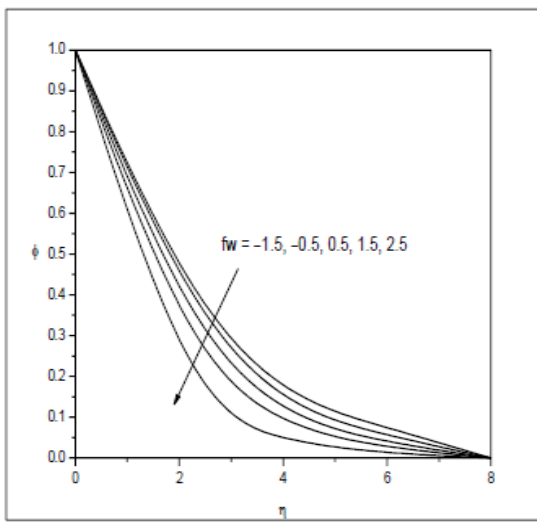

Fig. 33 Concentration profiles $(\phi)$ with $\mathrm{f}_{\mathrm{w}}$

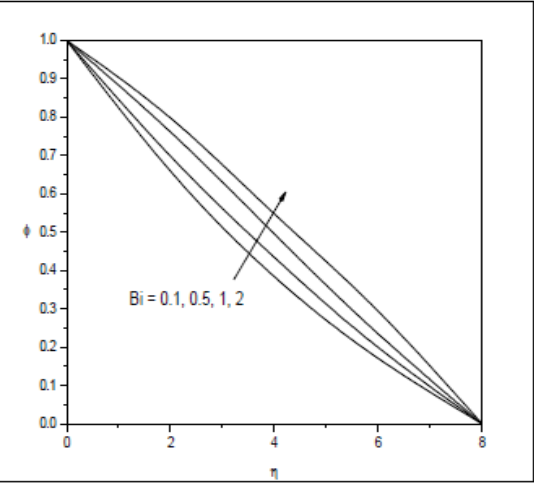

Fig. 31 Concentration profiles $(\phi)$ with $\mathrm{Bi}$

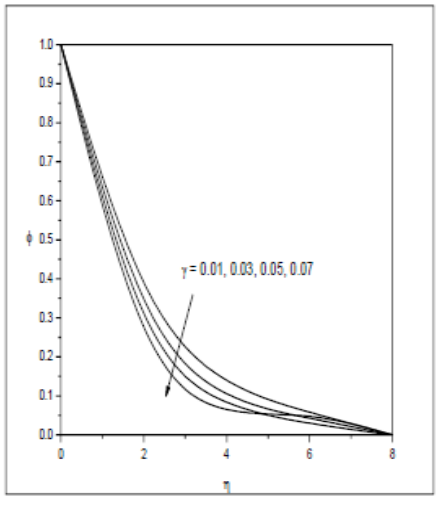

Fig. 34 Concentration profiles $(\phi)$ for different $\gamma$

TABLE2. Skin friction ( $\tau$ ), Nusselt Number (Nu) and Sherwood Number (Sh) at $\eta=0$

\begin{tabular}{|c|c|c|c|c|}
\hline \multicolumn{2}{|c|}{ Parameters } & $\tau(0)$ & $\mathrm{Nu}(0)$ & $\mathrm{Sh}(0)$ \\
\hline $\mathrm{N}$ & 1 & -1.5166 & 0.0175 & 0.4014 \\
& 2 & -1.3321 & 0.0221 & 0.4612 \\
& -0.5 & -1.5545 & 0.0165 & 0.4149 \\
& -0.8 & -1.6072 & 0.0201 & 0.6014 \\
\hline \multirow{3}{*}{$\mathrm{Kr}$} & 0.5 & -1.5166 & 0.0175 & 0.4034 \\
& 1.5 & -1.5281 & 0.0209 & 0.5139 \\
& -0.5 & -1.5097 & 0.0165 & 0.2981 \\
$\mathrm{Ec}$ & -1.5 & -1.4785 & 0.0099 & 0.1160 \\
\hline \multirow{3}{*}{$\mathrm{Q}$} & 0.01 & -1.5166 & 0.0175 & 0.4014 \\
& 0.03 & -1.5096 & 0.0765 & 0.3990 \\
& 0.05 & -1.4564 & 0.0801 & 0.3342 \\
\hline & 2 & -1.5166 & 0.0175 & 0.4014 \\
& 4 & -1.6080 & 0.0862 & 0.7923 \\
& -2 & -1.5242 & 0.4090 & 0.8162 \\
& -4 & -1.5997 & 0.6674 & 1.5664 \\
\hline
\end{tabular}


Effect of Newtonian Cooling and Non-Linear Density Variation on Non-Darcy Convective Heat and Mass Transfer

\begin{tabular}{|c|c|c|c|c|}
\hline $\mathrm{Sr} / \mathrm{Du}$ & $1.5 / 0.04$ & -1.5203 & 0.0213 & 0.4122 \\
& $1 / 0.06$ & -1.5191 & 0.0221 & 0.3996 \\
\hline \multirow{3}{*}{$\mathrm{Bi}$} & 0.1 & -1.5166 & 0.0175 & 0.4456 \\
& 0.3 & -1.5066 & 0.0399 & 0.4245 \\
& 0.5 & -1.4788 & 0.0453 & 0.3766 \\
\hline & 0.01 & -1.5166 & 0.0175 & 0.4456 \\
& 0.03 & -1.5209 & 0.0199 & 0.4234 \\
& 0.05 & -1.5312 & 0.0211 & 0.39876 \\
\hline
\end{tabular}

\section{CONCLUSION}

In this analysis we analyzed the effect of density ratio $(\gamma)$, Soret and Dufour effects ( $\mathrm{Sr} \& \mathrm{Du}$ ) on the flow characteristics using Runge-Kutta shooting technique. The important conclusions of this analysis are

- An increase in Grashof number (Gr) enhances the velocity. Concentration and reduces the temperature.

- smaller the permeability of the porous medium smaller the velocity and temperature while the concentration reduces with $\mathrm{M}$ and enhances with $\mathrm{Da}^{-1}$.

- The velocity and concentration reduces and temperature enhances in the flow region with increase in buoyancy ratio $(\mathrm{N}) .|\tau|$ and rate of mass transfer $(\mathrm{Sh})$ reduces on the wall with increase in $\mathrm{N}>0$ and enhances with $\mathrm{N}<0$. $|\mathrm{Nu}|$ enhances with $|\mathrm{N}|$ irrespective of the directions of the buoyancy forces.

- The velocity, temperature and concentration reduces in the degenerating chemical reaction case and in generating chemical reaction case we notice a depreciation in $\theta$ and $\phi$ and enhancement in $\mathrm{f}^{1}$ in entire fluid region. $|\tau|,|\mathrm{Nu}|$ and $|\mathrm{Sh}|$ enhances in the degenerating chemical reaction case and reduces in the generating chemical reaction case.

- An increase in the strength of heat generating /absorption reduces the velocity, temperature and concentration in the boundary layer. $|\tau|,|\mathrm{Nu}|,|\mathrm{Sh}|$ enhances with increase in the strength of heat generating / absorbing source.

- Higher the dissipative heat larger the temperature and smaller the velocity and concentration in the boundary layer. An increase in Eckert number $(\mathrm{Ec})$ reduces $|\tau|,|\mathrm{Sh}|$ and enhances $|\mathrm{Nu}|$ on the wall.

- An increase in the Soret parameter $\mathrm{Sr}$ (or decrease in the Dufour parameter Du) reduces the velocity, the temperature and concentration in the boundary layer. $|\tau|,|\mathrm{Nu}|$ and $|\mathrm{Sh}|$ enhances on the wall with increase in $\mathrm{Sr}$.

- An increase in convective heat transfer coefficient Bi leads to an enhancement in $\mathrm{f}^{1}, \theta$, and $\phi$. An increase in Bi reduces $|\tau|$, mass transfer and enhances the rate of heat transfer on the wall.

- An increase in Forchheimer number Fs reduces the velocity, temperature and concentration.

- An increase in the density ratio $\gamma$ reduces $\mathrm{f}^{1}, \theta$, and $\phi .|\tau|$ and $|\mathrm{Sh}|$ reduces and $|\mathrm{Nu}|$ enhances with $\gamma$.

\section{REFERENCES}

[1] Alam MS, Rahman MM,. Maleque MA, Ferdows M, "Dufour and Soret effects on steady MHD combined free-forced convective and mass transfer flow past a semi-Infinite vertical plate", Thammasat, Int. J. Sci. Technol., 2006, 11(2), 1-12.

[2] Alam MS, Rahman MM, "Dufour and soret effects on MHD free convective heat and mass transfer flow past a Vertical Porous flat plate embedded in a Porous Medium", J. Naval Architecture and Marine Engineering, 2005, 2(1), 55-65.

[3] Anghel M, Takhar HS and Pop I, "Dufour and Soret effects on free convection boundary layer over a vertical surface embedded in a porous medium", Stud. Univ. Babes-Bolyai, Math., 2000, 45, 11-21.

[4] Anjali Devi S P and David A M G, "Effects of variable viscosity and nonlinear radiation on MHD flow with heat transfer over a surface stretching with a power-law velocity", Advances in Applied Science Research, 2012, 3(1), 319-334.

[5] Chamkha AJ, Ben-Nakhi A, "MHD mixed convection-radiation interaction along a permeable surface immersed in a porous medium in the presence of Soret and Dufour effects", Heat Mass Transfer, 2008, 44(7), 845-856. 
[6] Chaudary R.C and Jain P, "An exact solution to the un-steady free- convection boundary-layer flow past an impulsively started vertical surface with Newtonian heating", J.Eng. Phys.Thermophys, 2007, 80, 954-960.

[7] Gangadhar K, "Soret and Dufour Effects on Hydro Magnetic Heat and Mass Transfer over a Vertical Plate with a Convective Surface Boundary Condition and Chemical Reaction", Journal of Applied Fluid Mechanics, 2013, 6(1), 95-105.

[8] Goren, S.L, “On free convection in water at $4^{\circ} \mathrm{C}^{\prime}$, Chem. Engg. Sci, 1966, 21, 515.

[9] Govindarajulu,"Free Convection flow of water at $40 \mathrm{C}$ on vertical and horizontal plate”, Chem. Engg. Sci., 1970, 25, 18-27.

[10] Hossain MA, Molla MM, Yaa LS, "Natural convection flow along a vertical wavy surface with uniform surface temperature in presence of heat generation/absorption", Int. J.Thermal Science, 2004, 43, 157-163.

[11] Kishan N and Srinivas M, "Thermophoresis and viscous dissipation effects on Darcy-Forchheimer MHD mixed convection in a fluid saturated porous media", Advances in Applied Science Research, 2012,3(1), 6074.

[12] Lavanya B, Leela Ratnam A, "Dufour and Soret effects on steady MHD free convective flow past a vertical porous plate embedded in a porous medium with chemical reaction heat generation and viscous dissipation", Advances in Applied Science Research, 2014, 5(1), 127-142.

[13] Lesnic D, Ingham D. B, Pop I and Storr C, “Free convection boundary layer flow above a nearly horizontal surface in a porous medium with Newtonian heating", Heat and Mass Transfer, 2004, 40, 665-672.

[14] Madhusudhana Rao B, Vishwanatha Reddy G,"Soret and Dufour effects on Hydro-Magnetic heat and mass transfer over a vertical plate in a porous medium with a convective surface boundary condition and chemical reaction", Int journal of Engg Research and Applications, 2012, 2(4), 56-76.

[15] Merkin J. H, "Natural convection boundary-layer flow on a vertical surface with Newtonian heating", Int. J.Heat fluid flow, 1994, 15, 392-398.

[16] Mohammed Ibrahim S, "Chemical reaction and radiation effects on MHD free convection flow along a stretching surface with viscous dissipation and heat generation", Advances in Applied Science Research, 2013, 4(1), 371-382.

[17] Mohammed Ibrahim S, Bhaskar Reddy N, "Radiation and Mass transfer effects on MHD free convection flow along a stretching surface with viscous dissipation and heat generation", Int. J. of Appl. Math and Mech, 2011, 8(8), 1-21.

[18] Postelnicu A, "Influence of a magnetic field on heat and mass transfer by natural convection from vertical surfaces in porous media considering Soret and Dufour effects", Int. J. Heat Mass Transfer, 2004, 47, 1467-1472.

[19] Rajasekhar K., Ramana Reddy G V and Prasad B D C N, "Chemically Reacting on MHD Oscillatory Slip Flow in a Planer Channel with Varying Temperature and Concentration", Advances in Applied Science Research, 2012, 3(5), 2652-2659.

[20] Rajesh V and Chamkha Ali J. "Unsteady convective flow past an exponentially accelerated infinite vertical porous plate with Newtonian heating and viscous dissipation", Int. Journal of Numerical Methods for Heat \& Fluid Flow, 2014, 24(5), 1109-1123.

[21] Vajravelu K, Hadjinicolaou A," Heat transfer in a viscous fluid over a stretching sheet with viscous dissipation and internal heat generation", Int. Comm. Heat Mass. 20, 417-430 (1993).

\section{AUTHOR'S BIOGRAPHY}

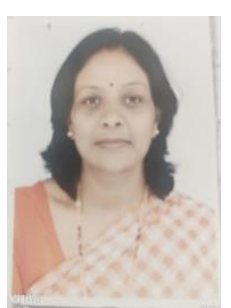

Prof. Anjna Singh, Professor, Department of Mathematics, Government Girls P.G. College, Rewa-486001, Madhya Pradesh, India and had got M.Phil. Degree in 19891990 from Arga University, Agra, India and Ph.D. Degree in July 2002 from Dr.Bhim Rao Ambedkar University, Agra, India. She has lot of teaching experience as Assistant Professor from 1993-98, Senior Assistant Professor from 1998-2003, Associate Professor from 2003-2006, as a Professor since from 2007 till-to-date. She has guided research work to 5 students under her supervision. Her area of research work is Fluid Dynamics. She has attended National and International Workshops and Seminars.

Citation: Anjna Singh., (2019). Effect of Newtonian Cooling and Non-Linear Density Variation on NonDarcy Convective Heat and Mass Transfer. International Journal of Scientific and Innovative Mathematical Research (IJSIMR), 7(6), pp.1-11. http://dx.doi.org/10.20431/2347-3142.0706001

Copyright: (C) 2019 Authors, this is an open-access article distributed under the terms of the Creative Commons Attribution License, which permits unrestricted use, distribution, and reproduction in any medium, provided the original author and source are credited. 